Werten über $260 \mathrm{mg} / \mathrm{dl}$ ist die Insulingabe unumgänglich “, so Sippel. Bei den OAD sind Sulfonylharnstoffe wegen des Risikos nächtlicher Hypoglykämien nicht empfehlenswert. Auch Glitazone sind wegen ihres verzögerten Wirkeintritts nicht sinnvoll. Eingesetzt werden können Glinide, die aber nur kurz wirken, Metformin, das die hepatische Glukoneogenese hemmt, und besonders Gliptine. „Mit letzteren können die postprandialen BZSpitzen am effektivsten behandelt werden“, so Sippel. Sei eine Kombination indiziert, so könne Metformin mit einem Gliptin, Glinid oder Inkretinmimetikum kombiniert werden. Dagegen ist Metformin plus Sulfonylharnstoff weniger sinnvoll. Gleiches gilt für die basal unterstützte orale Therapie, da der NüchternBZ morgens beim Steroiddiabetes meist nicht stark erhöht sind.

\section{Besonderheiten der Insulintherapie}

Ist eine Insulintherapie indiziert, so empfiehlt sich die dreimalige prandiale Gabe von Normalinsulin oder ein Mischinsulin, wobei die kalkulierte Insulin-Tagesdosis bei 0,15-0,3 IE pro kg KG liegt. Eine vorbestehende Diabetestherapie ist anzupassen (Tab. 1). Bei einem Patienten von $80 \mathrm{~kg}$, bei dem bisher kein Diabetes bekannt war, ist entweder die Gabe von 6-6-4 IE Normalinsulin oder 12-24 IE eines 30/70 oder 50/50 Mischinsulins morgens ratsam. „Nicht sinnvoll beim Steroiddiabetes ist die Gabe eines kurz wirksamen Analoginsulins, da es nicht lange genug wirkt", so Sippel. Die Insulindosis müsse auftitriert werden. Nur bei erhöhten Nüchtern-BZ-Werten morgens sei eventuell abends zusätzlich ein Misch- bzw. Basalinsulin sinnvoll. Wird im Verlauf die Steroidtherape ausgeschlichen, so muss die Insulindosis ebenfalls reduziert werden. „Die prozentuale Dosisreduktion des Steroids erfordert die gleiche prozentuale Reduktion beim Insulin“, so Sippel. Dr. med. PeterStiefelhagen, Hachenburg

Quelle: Innere Medizin fachübergreifend - Diabetologie grenzenlos, 1.3.2013 in München

„Ein System kollabiert"

\title{
Ärztliche Weiterbildung in akuter Gefahr?
}

\author{
Mehr Fälle pro Arzt, mehr Erlöse pro Bett, Bonusverträge für Chefärzte statt Berufsethos: Der \\ BDI-Vize von Römer beklagt die Kommerzialisierung im Krankenhaus und sieht dort die ärztliche \\ Weiterbildung akut gefährdet.
}

Beim Münchner Kongress „Innere Medizin fachübergreifend Diabetologie grenzenlos“, kam der BDI-Vize in Fahrt: „Unter den Bedingungen des DRG-Systems und der forcierten Durchsetzung ökonomischer Ziele“, so von Römer, „wandeln sich die Krankenhäuser in Deutschland vielerorts von Orten der sozialen und medizinischen Vor- und Fürsorge zu Unternehmen, in denen Krankheit zur Ware und Ärzte zu Anbietern werden“.

Von Römer (Abb. 1) geißelte die Bonusverträge der Chefärzte: Ärzte würden damit animiert, Unternehmensziele über das $\mathrm{Pa}$ tientenwohl zu stellen und Leistungen anzubieten, die v.a. dem Krankenhaus nutzen. Viele Kollegen kommen so in Schwierigkeiten mit Berufsethik und Berufsrecht, beklagte von Römer.

\section{Eklatanter Personalmangel}

Eine weitere Folge der Kommerzialisierung sei der Personalmangel in den Krankenhäusern. Pflegekräfte eingerechnet, feh-

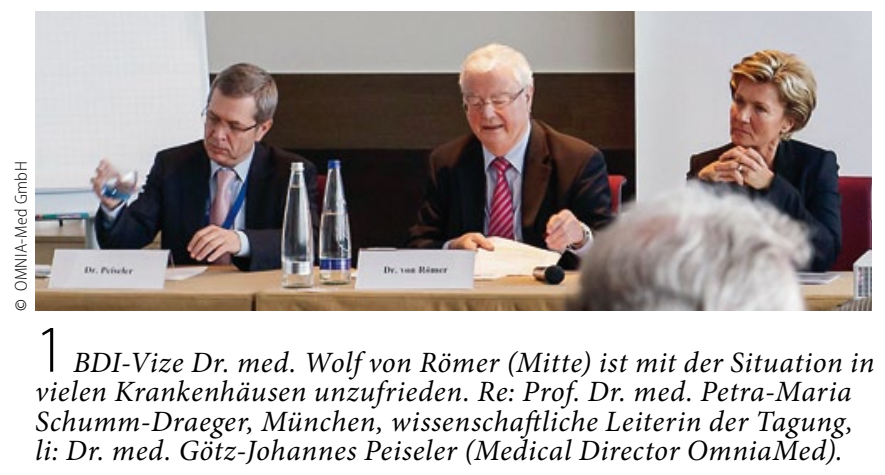

len in Deutschlands Krankenhäusern etwa 160000 Vollzeitstellen. Das Personal arbeite am Limit und schiebe bundesweit eine Million Überstunden vor sich her. Statt am Personal zu sparen, um die Klinik auf Wirtschaftlichkeit zu trimmen, sei ein Personalaufbau von Nöten. Gründe sind die Zunahme multimorbider Patienten durch die demografische Entwicklung sowie sich verändernde Rahmenbedingungen wie die Auswirkungen des Arbeitszeitgesetzes, aufwendige Qualitätssicherungsmaßnahmen und ausufernde Bürokratie.

Vor diesem Hintergrund unterstützt von Römer die Forderung der Gewerkschaft Verdi nach gesetzlicher Regelung der Personalbemessung.

\section{Arztberuf immer unattraktiver}

Eine weitere Folge des Personalmangels: „Chef-, Ober- und Fachärzte werden in den Funktionsabteilungen absorbiert und stehen in nicht ausreichendem Maße für die ärztliche Aus- und Weiterbildung zur Verfügung“, so von Römer. Defizite in der Weiterbildung führen zur Abnahme der Versorgungsqualität und der Patientensicherheit. Darüber hinaus, so von Römer, machen sie den Arztberuf für jüngere Kollegen unattraktiv.

Deshalb forderte von Römer, die Krankenhausträger zu verpflichten, die ärztliche Weiterbildung zu garantieren. Die Ärztekammern rief er auf, die Weiterbildungsordnung „sinnvoll zu überarbeiten und zu verschlanken“. Dr. med. Dirk Einecke, München grenzenlos", München, 01.03.2013 (Veranstalter: Berufsverband Deutscher Internisten e.V. (BDI), OMNIA-Med GmbH) 